\title{
UMM-EL-BREIGÂT (TEBTYNIS) 2015: LE DUE CANTINE DEI PAPIRI E I DUE TEMPLI DI SOKNEBTYNIS
}

\author{
Nota del s.c. CLAUDIO GALLAZZI (*)
}

(Adunanza del 4 febbraio 2016)

SunTO. - Poco prima che iniziasse la sua campagna a Tebtynis dell'anno 2015, la Missione archeologica franco-italiana, che là opera dal 1988, ha avuto a sua disposizione il quaderno di scavo di Carlo Anti relativo ai lavori svolti nel sito dal 1930 al 1932. Seguendo le indicazioni fornite dal quaderno, la Missione ha potuto identificare, all'interno del santuario di Soknebtynis, le due cantine che avevano dato un'enorme quantità di papiri nel 1931 e la cui ubicazione era rimasta ignota. Successivamente la Missione ha scoperto il tempio eretto all'epoca di Tolomeo Soter al di sotto di quello individuato nel 1899 e scavato tra il 1931 e il 1933, che risale al I sec. a.C., non all'inizio dell'età tolemaica, come si riteneva.

$* * *$

RÉsumé. - Juste avant d'entreprendre sa campagne 2015 à Tebtynis, la Mission archéologique franco-italienne, qui est à l'œuvre sur le site depuis 1988, a eu à sa disposition le cahier de fouille de Carlo Anti concernant les travaux réalisés sur les lieux de 1930 à 1932. Grâce aux indications du cahier, la Mission a pu repérer à l'intérieur du sanctuaire de Soknebtynis les deux caves dans lesquelles une quantité très importante de papyrus fut récoltée en 1931 et dont l'emplacement était demeuré inconnu. Ensuite, l'équipe a découvert le temple érigé à l'époque de Ptolémée Soter sous les vestiges du sanctuaire retrouvé en 1899 et dégagé en 1931-1933, qui doit être daté du Irr s. av. J.-C. et non, comme tout le monde le pensait, du début de l'époque ptolémaïque.

(") Università degli Studi di Milano; Dipartimento di Studi Letterari, Filologici e Linguistici; Centro di Papirologia «Achille Vogliano», Milano, Italy.

E-mail: claudio.gallazzi@unimi.it 
Nel novembre del 2014, alla conclusione dei lavori svolti sul kôm di Umm-el-Breigât, dove opera dal 1988 fra le rovine dell'antica Tebtynis, ${ }^{1}$ la Missione archeologica franco-italiana costituita dall'Institut français d'archéologie orientale del Cairo e dall'Università degli Studi di Milano, aveva previsto di svolgere la successiva campagna negli stessi settori in cui scavava da anni, cioè nel quartiere dell'abitato a nord-ovest del santuario di Soknebtynis ${ }^{2}$ e nella discarica situata ad est del medesimo luogo di culto. ${ }^{3}$ Come era stato programmato, nel set-

1 Per i lavori eseguiti e i risultati ottenuti dal 1988 al 2001 si rimanda alla bibliografia citata in C. Gallazzi, Tebtynis (Umm-el-Breigât - Fayûm), RISE 1 (2004), 115-127, in partic. 123, nt. 3, cui si aggiunga G. Hadji-Minaglou, Tebtynis, IV. Les habitations à l' est du temple de Soknebtynis, Le Caire 2007. Per l'attività svolta negli anni seguenti si vedano almeno C. Gallazzi, Umm-el-Breigât (Tebtynis): 2002, ASAE 79 (2005), 107-114; Id., Tebtynis (Umm-el-Breigât - Fayûm). Campagne di scavo 2003 e 2004, RISE 2 (2006), 177 196; Id., Umm-el-Breigât (Tebtynis) 2004-2008: gli scavi nel settore bizantino, RIL 144 (2010), 183-208; Id., Umm-el-Breigât (Tebtynis). Campagne di scavo 2009-2010, RISE 5 (2011), 109-124; Id., Umm-el-Breigât (Tebtynis). Campagna 2011, RISE 6 (2013), 141-156; Id., Umm-el-Breigât (Tebtynis): campagna di scavo dell'anno 2012, RIL 146 (2012), 87-110; C. Gallazzi - G. Hadji-Minaglou, Umm-el-Breigât (Tebtynis), in Rapport d'activité 20132014, Suppl. BIFAO 114 (2014), 76-79; Iid., Umm-el-Breigât (Tebtynis), in Rapport d'activité 2014-2015, Suppl. BIFAO 115 (2015), 82-90.

2 In tale area la Missione ha lavorato dal 1996 al 1999 e dal 2009 in poi: cfr. C. Gallazzi, Due campagne di scavo a Umm-el-Breigât (Tebtynis): 1995 e 1996, Acme 50. III (1997), 15-30, in partic. 20-28; Id., I lavori a Umm-el-Breigât (Tebtynis) degli anni 1997. 1999, Acme 55. I (2002), 3-31, in partic. 5-26; Id., Umm-el-Breigât (Tebtynis). Campagne di scavo 2009-2010 cit. (nt. 1), 110-117; Id., Umm-el-Breigât (Tebtynis): campagna di scavo dell'anno 2012 cit. (nt. 1), 89-104; Gallazzi - Hadji-Minaglou, Umm-elBreigât (Tebtynis), in Rapport d'activité 2013-2014 cit. (nt. 1), 76-79; Iid., Umm-elBreigât (Tebtynis), in Rapport d'activité 2014-2015 cit. (nt. 1), 82-87.

3 L'aspetto del monticolo di pattume, il modo in cui si è formato e i risultati degli scavi in esso compiuti negli anni immediatamente successivi alla scoperta del 1994 sono illustrati in C. Gallazzi, Lo scavo di una discarica a Umm-el-Breigât (Tebtynis), ovvero, le sorprese del pattume, NAC 27 (1998), 185-207. I lavori effettuati nelle campagne seguenti sono rapidamente descritti in Gallazzi, I lavori cit. (nt. 2), 28-31; B. Mathieu, Travaux de l'Institut français d'archéologie orientale en 2000-2001, BIFAO 101 (2001), 449-610, in partic. 552-553; Id., Travaux de l'Institut français d'archéologie orientale en 2001-2002, BIFAO 102 (2002), 437-614, in partic. 530; Gallazzi, Tebtynis (Umm-elBreigât - Fayûm) cit. (nt. 1), 121-122; Id., Umm-el-Breigât (Tebtynis): 2002 cit. (nt. 1), 109; Id. Tebtynis (Umm-el-Breigât-Fayûm). Campagne di scavo 2003 e 2004 cit. (nt. 1), 180-182; Id., Umm-el-Breigât (Tebtynis). Campagna di scavo 2005, RISE 3 (2009), $97-$ 107, in partic. 102-103; Id., Umm-el-Breigât (Tebtynis). Campagne di scavo 2006-2008, RISE 4 (2010), 121-141, in partic. 130-133; Id. Umm-el-Breigât (Tebtynis). Campagne di 
tembre del $2015 \mathrm{i}$ lavori sono stati intrapresi fra gli edifici a nord-ovest del tempio e sul monticolo del pattume e sono proseguiti regolarmente sino all'inizio di novembre. Sia in un settore sia nell'altro lo scavo ha dato gli esiti previsti, consentendo di rimettere alla luce costruzioni rilevanti, di acquisire nuove conoscenze su un quartiere a vocazione commerciale e artigianale e di recuperare una discreta quantità di testi su papiri e cocci scritti in ieratico, demotico e greco. ${ }^{4}$

Tuttavia, i risultati più significativi della stagione sono stati raccolti in maniera inaspettata, non tra le case dell'abitato o fra i detriti della discarica, ma all'interno del santuario di Soknebtynis, in cui la Missione, alla fine del '14, non aveva previsto intervento alcuno. Il luogo di culto, infatti, era stato scoperto nel 1899 da Bernard Grenfell e Arthur Hunt, che ne avevano misurato il mastodontico peribolo, ne avevano scavato alcuni ambienti ed avevano constatato che il noos in pietra era stato completamente smantellato in età bizantina ed araba. ${ }^{5}$ Successivamente, fra il 1931 e il 1933, Carlo Anti e Gilberto Bagnani avevano scavato la struttura, rimettendola alla luce per intero, e ne avevano attribuito la fondazione a Tolomeo Soter, mentre avevano datato la maggior parte degli edifici del temenos alla seconda metà del periodo tolemaico e all'epoca romana. ${ }^{6}$ Dopo Anti e Bagnani, la nostra Missione aveva anch'essa operato una prima volta nel tempio fra il 1990 e il 1993, effettuando rilievi, sondaggi e studi, che hanno trovato una conveniente presentazione in Rondot, Tebtynis, II. Le temple cit. (nt. 6). Si era poi tornati a lavorare nel santuario

scavo 2009-2010 cit. (nt. 1), 117-120; Id., Umm-el-Breigât (Tebtynis): campagna di scavo dell'anno 2012 cit. (nt. 1), 105-110; Gallazzi - Hadji-Minaglou, Umm-el-Breigât (Tebtynis), in Rapport d'activité 2014-2015 cit. (nt. 1), 87-90.

4 Una prima presentazione del lavoro svolto e dei risultati acquisiti è stata fatta in C. Gallazzi - G. Hadji-Minaglou, Umm-el-Breigât (Tebtynis), in Rapport d'activité 2015-2016, Suppl. BIFAO 116 (2016), 70-81.

5 Cfr. B.P. Grenfell - A.S. Hunt, A large find of Ptolemaic Papyri, APF 1 (1901), 376-378, in partic. 376.

6 Cfr. C. Anti, Gli scavi della Missione archeologica italiana a Umm el Breighât (Tebtunis), Aegyptus 11 (1930-1931), 389-391; Id., Scavi a Tebtunis, in Atti del IV Congresso internazionale di papirologia, Milano 1936, 473-478, in partic. 474-475; G. Bagnani, Gli scavi di Tebtunis, Aegyptus 14 (1934), 3-13, in partic. 5-7. Si veda, inoltre, la pianta del tempio tracciata da Fausto Franco negli anni Trenta e riprodotta in V. Rondot, Tebtynis, II. Le temple de Soknebtynis et son dromos, Le Caire 2004, Plan 2, dove i vari edifici sono distinti cromaticamente in base alla data ad essi assegnata.

7 Cfr. Rondot, Tebtynis, II. Le temple cit. (nt. 6), 7-8. 
alla fine del 2011, rimuovendo la sabbia dalla porta d'ingresso dissepolta nel 1931 e scoprendo al di sotto di questa i resti di un pilone e di un portale più antichi. ${ }^{8}$ Quindi nel novembre del 2014 non c'era alcuna ragione pressante, perché la missione, già impegnata in un'ampia attività di scavo, programmasse pure dei lavori nel tempio per la stagione successiva.

Però, nella tarda primavera del 2015 il quaderno di scavo di Carlo Anti, relativo alle campagne svolte a Tebtynis dal 1930 al 1932, è stato messo a disposizione della Missione. ${ }^{9}$ In tale taccuino è ovviamente descritta la mirabile scoperta di due cantine piene di papiri, che fu realizzata da Anti nel tempio il 10 marzo 1931. Dai due ripostigli furono estratte centinaia di testi in geroglifico, ieratico, demotico e greco, talvolta lunghi qualche metro, più frequentemente ridotti a frammenti, che erano stati buttati là dentro come roba da bruciare, ma che fortunatamente non erano stati dati alle fiamme. Era materiale appartenente ad un enorme insieme di papiri connessi col santuario ed il suo clero, che è attualmente conosciuto come "deposito del tempio di Soknebtynis". ${ }^{10}$ Una parte del lotto fu raccolta all'interno del santuario o in prossimità di esso da Grenfell e Hunt (1899-1900), da Otto Rubensohn (1902), da scavatori locali (fine anni Venti), da Achille Vogliano (1934) e dalla nostra Missione, mentre la porzione più cospicua fu rinvenuta da Anti nelle cantine citate. ${ }^{11}$ Purtroppo, né Anti né il

8 Cfr. Gallazzi, Umm-el-Breigât (Tebtynis). Campagna 2011 cit. (nt. 1), 144148; C. Gallazzi - G. Hadji-Minaglou, Umm-el-Breigât (Tebtynis), in Rapport d'activité 2011-2012, Suppl. BIFAO 112 (2012), 71-77.

9 Il taccuino è attualmente depositato a Mestre, presso gli archivi dell'Istituto Veneto di Scienze, Lettere ed Arti, al cui Presidente, prof. Gherardo Ortalli, ed ai cui Organi Direttivi esprimo la più sentita gratitudine per avermi consentito l'accesso al manoscritto e per averne autorizzata la pubblicazione nella serie monografica della Missione Fouilles franco-italiennes. Tebtynis.

10 Benché solo una parte minoritaria dei reperti del "deposito" sia stata pubblicata, è impossibile citare qui tutte le edizioni dei pezzi fatti conoscere; pertanto si rinvia a K. Ryholt, On the Contents and Nature of the Tebtynis Temple Library, in S. Lippert - M. Schentuleit (hg.), Tebtynis und Soknopaiu Nesos im römerzeitlichen Fajum. Akten des Internationalen Symposions von 11. bis 13. Dezember in Sommerbausen bei Würzburg, Wiesbaden 2005, 141-170, dove sono elencate le varie categorie di testi rappresentate nel lotto di materiale. Si rimanda, invece, a C. Gallazzi, I papiri del tempio di Soknebtynis: chi li ha trovati, dove li hanno trovati, in C. Gallazzi (ed.), Tebtynis, VI. Scripta varia, I, Le Caire 2018, 113-179, in partic. 147-157, per un'individuazione dei luoghi e un'indicazione degli anni, in cui i papiri del gruppo furono recuperati.

11 Gallazzi, I papiri del tempio cit. (nt. 10), 147-149. 
suo collaboratore Bagnani pubblicarono mai informazioni dettagliate sul ritrovamento, non stamparono fotografie e non diedero una pianta della costruzione, in cui il materiale era stato raccolto. ${ }^{12}$ Conseguentemente si sapeva che i papiri erano stati recuperati in due ambienti sotterranei posti nella parte orientale del temenos; ma si ignorava sotto quale edificio le due cantine fossero situate. In verità, nel corso degli anni erano state formulate varie ipotesi sull'ubicazione dei ripostigli, ma nessuna di esse era convincente. Non poteva essere accolta l'idea espressa in J. Osing, The Carlsberg Papyri, 2. Hieratische Papyri aus Tebtunis, I, Copenhagen 1998, 19, che le cantine si trovassero fra il lastricato del dromos e il lato occidentale del muro di cinta del tempio, perché Anti, Bagnani e Botti dicono tutti che gli ambienti stavano nel settore orientale del temenos. Analogamente infondata era l'ipotesi avanzata in Rondot, Tebtynis, II. Le temple cit. (nt. 6), 31 che i papiri fossero stati rinvenuti sotto la stanza indicata col nr. 36A sulla pianta del tempio stilata da Franco, menzionata sopra a nt. $6 ;^{13}$ sotto

12 Anti menzionò rapidamente la scoperta a p. 391 dell'articolo di Aegyptus 11 citato a nt. 6, il quale è stampato pure in Bollettino dell'Associazione di studi mediterranei 2. II (1931), 23-24 e in Atti Ven. 91 (1931-1932), 1183-1187; e ne fece parola anche sulla prima pagina del Marzocco del 31 maggio 1931, senza però fornire dettagli aggiuntivi. Più prodigo di particolari, impressioni personali e note di colore fu, invece, Bagnani in una lettera alla moglie scritta il giorno dopo la scoperta e riprodotta in D.J.I. Begg, "It was Wonderful, Our Return in the Darkness with... the Baskets of Papyri! Papyrus Finds at Tebtunis from Bagnani Archives, 1931-1936, BASP 35 (1998), 185-210, in partic. 189-191; ma pure lui fu vago sul luogo del ritrovamento, accennando genericamente a «some houses on the east wall of the temenos» e a «two small cellars side by side». Non disse di più Giuseppe Botti, che per primo studiò i papiri ieratici e demotici raccolti, allorché riferì della scoperta in I papiri ieratici e demotici degli scavi italiani di Tebtynis, in Atti del IV Congresso internazionale di papirologia, Milano 1936, 217-223, in partic. 219, e in Quello che anche l'Egittologia deve a Carlo Anti, in "Anthemon", scritti di Archeologia e di Antichità Classiche in onore di Carlo Anti, Venezia 1954, 3-8, in partic. 3, poi in Omaggio a Giuseppe Botti, Milano 1984, 33-38, in partic. 33.

13 La supposizione è ripresa in I. Guermeur, Les nouveaux papyrus hiératiques exhumés sur le site de Tebtynis: un aperçu, in S. Lippert - M. Schentuleit (eds.), GraecoRoman Fayum - Texts and Archaeology, Proceedings of the Third International Fayum Symposion, Freudenstadt, May 29 - June 1, 2007, Wiesbaden 2008, 113-122, in partic. 113, ed in T.M. Hickey, Tebtunis on the Arno (and) Beyond: Two "Archives", in G. Bastianini - A. Casanova (edd.), 100 anni di istituzioni fiorentine per la Papirologia. Atti del convegno internazionale di studi. Firenze, $12-13$ giugno 2008, Firenze 2009, 67-81, in partic. 70 , nt. 13 . 
tale ambiente, infatti, è posta un'unica cantina, non due, come scrivono Anti e i suoi collaboratori e come si vede nell'unica fotografia dei ripostigli conservata nell'archivio di Anti presso l'Università di Padova (Fig. 1). ${ }^{14}$ Utilizzando proprio quell'immagine come riferimento, nel settembre del 2010 la nostra Missione aveva cominciato i suoi tentativi di localizzare i ripostigli fra i ruderi del temenos ormai sepolti quasi del tutto sotto cumuli di sabbia depositata dal vento; ma, nonostante l'impegno profuso, nessun risultato era stato ottenuto. Le ricerche furono riprese l'anno seguente e furono concentrate nel settore nord-orientale del tempio. Là l'obiettivo parve finalmente raggiunto una dozzina di metri a nord della porta laterale del temenos: sotto l'ambiente indicato come 27B sulla pianta di Franco si erano individuate due cantine affiancate e coperte da volte a botte, che sembravano quelle riprodotte nella fotografia di Anti. Le apparenti somiglianze con l'immagine del 1931 e la collocazione della stanza sul lato est del tempio indussero a identificare i due ripostigli sottostanti a 27B come il luogo della scoperta del 10 marzo 1931, tanto che se ne diede notizia in Gallazzi Hadji-Minaglou, Umm-el-Breigât (Tebtynis), in Rapport d'activité 20112012 cit. (nt. 8), 72-73, ed in Gallazzi, Umm-el-Breigât (Tebtynis). Campagna 2011 cit. (nt. 1), 144-146. Tale identificazione, tuttavia, è apparsa priva di fondamento, allorché si è avuto fra le mani il quaderno di Anti, in cui è scritto che le due cantine stavano sotto l'edificio contrassegnato col nr. 32. Per questa ragione, nei giorni iniziali della campagna del 2015 si è deciso di apportare una lieve variazione al programma di lavoro stabilito e di inviare una piccola squadra nel temenos per localizzare le cantine, sgombrarle dalla sabbia e fotografarle. Si riteneva che con questo intervento limitato e poco impegnativo si sarebbe finalmente risolto il problema dell'ubicazione dei ripostigli e si sarebbero avute alcune immagini da inserire nel vol. VI della serie Fouilles franco-italiennes. Tebtynis, ormai in corso di stampa, molte pagine del quale erano dedicate ai papiri del "deposito del tempio" e al ritrovamento di Anti.

14 Negativo e stampa della fotografia, precedentemente conservati presso il Dipartimento di Archeologia, ora sono custoditi presso il Dipartimento di Beni Culturali dell'Ateneo patavino, alla cui Direzione ed al cui Personale manifesto la mia riconoscenza per la cortesia, con cui hanno messo il materiale a mia disposizione e hanno agevolato il mio lavoro su di esso. 


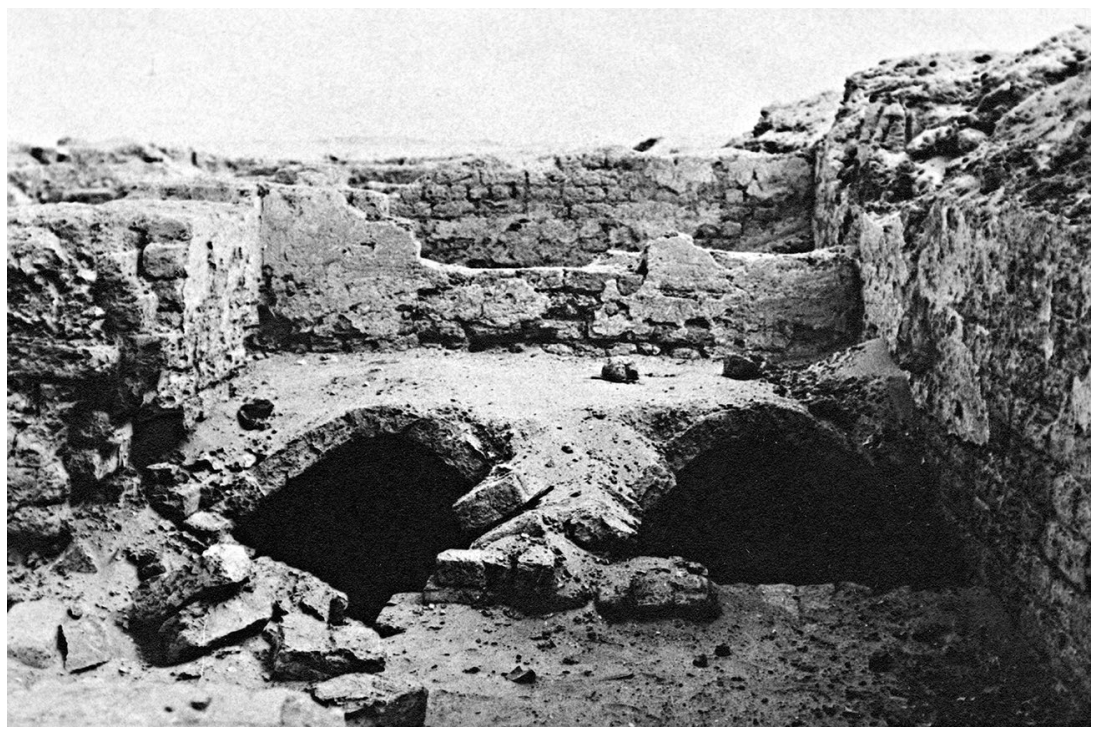

Fig. 1 - Fotografia delle due cantine scattata nel 1931.

Incominciati i lavori, via via che la sabbia era rimossa ed i ruderi affioravano, l'edificio 32 si rivelava assai più deteriorato rispetto al momento della scoperta, sia per i guasti provocati dall'erosione eolica, sia per le devastazioni prodotte da saccheggiatori locali intervenuti successivamente agli scavi di Anti. ${ }^{15}$ La parete sud della costruzione era completamente scomparsa; la facciata ovest era ridotta a due corsi di mattoni della metà settentrionale e il muro nord conservava appena una parte della fondazione ad ovest ed uno spuntone corroso nel mezzo; mentre le strutture centrali della costruzione erano divenute un cumulo di mattoni divelti. Il battuto dei pavimenti era stato rimosso pressoché dovunque ed anche le cantine nel sottosuolo erano state danneggiate: la colmatura al di sopra delle volte era stata quasi tutta asportata; il muro di chiusura a nord era stato abbattuto pressoché per intero e quello a sud aveva perso la metà superiore; la volta del ripostiglio ad ovest mancava di molti mattoni,

15 Della localizzazione delle cantine si tratta pure in Gallazzi, I papiri del tempio cit. (nt. 10), 141-146, e Gallazzi - Hadji-Minaglou, Umm-el-Breigât (Tebtynis), in Rapport d'activité 2015-2016 cit. (nt. 4), 78-82. 
soprattutto nel tratto centrale; quella del ripostiglio orientale, invece, era rimasta pressoché inalterata, ma in corrispondenza della botola di accesso i saccheggiatori avevano scavato un buco profondo quasi $1 \mathrm{~m}$. dentro l'adiacente muro di cinta del temenos (Fig. 2).

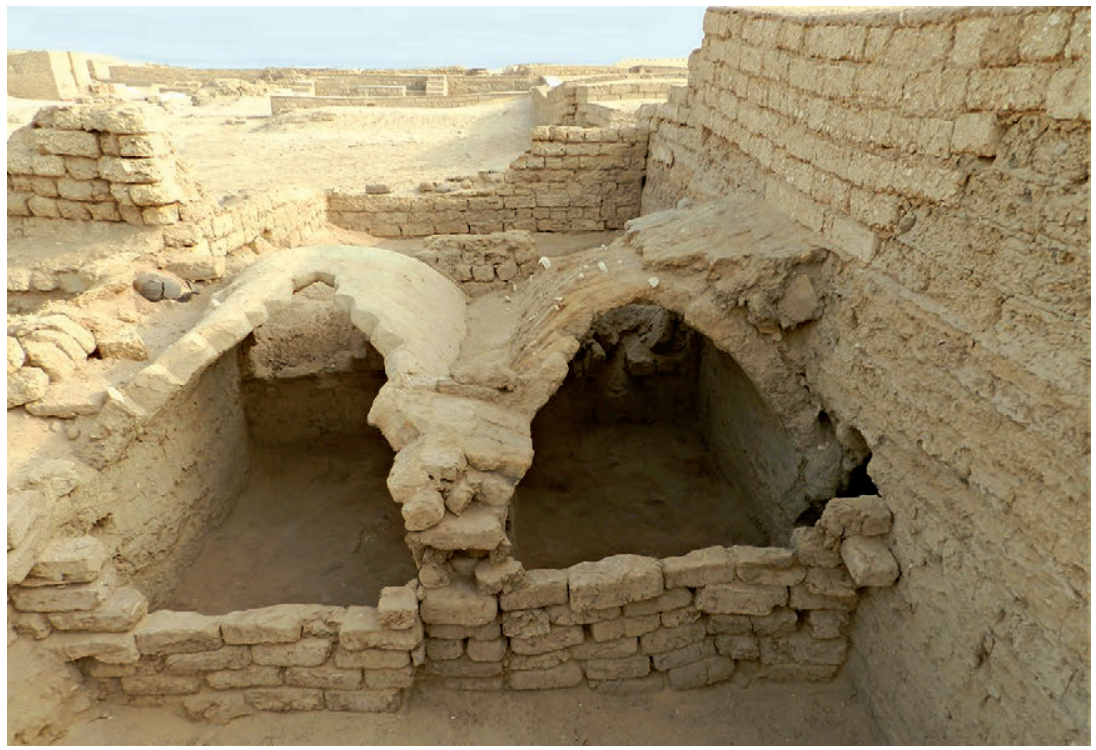

Fig. 2 - Le due cantine nel settembre del 2015.

Nonostante questi danni, i ruderi rimasti ed il rilievo eseguito da Franco durante gli scavi di Anti hanno permesso di riconoscere nell'edificio uno dei tanti pastophoria, cioè degli alloggi assegnati ai membri del clero, che erano addossati al peribolo del tempio. ${ }^{16} \mathrm{Al}$ pari delle costruzioni analoghe dislocate dentro il temenos, anche 32 fu edificato a contatto diretto col peribolo, evitando di erigere la parete adiacente alla muraglia, vale a dire quella ad est. Il pastophorion fu impiantato su di una base

16 Su tali costruzioni si vedano U. Wilcken, Urkunden der Ptolemäerzeit, I, Berlin - Leipzig 1927, 46; G. Husson, OIKIA. Le vocabulaire de la maison privée en Égypte d'après les papyrus grecs, Paris 1983, 221-223; S.E. Thomas, The Pastophorion: 'Priest's Houses' in Legal Texts from Ptolemaic Pathyris and Elsewhere in Egypt, JEA 99 (2013), 155-169; Id., The Pastophorion Revisited: Owners and Users of the 'Priest's Houses' in Ptolemaic Pathyris and Elsewhere in Egypt, JEA 100 (2014), 111-132. 
rettangolare di $\mathrm{m} .3,30 \times 6,80^{17}$ e comprendeva due stanze: una ad ovest di m. 2,30 x 2,80, l'altra ad est di m. 2,30 × 3 (Fig. 3). ${ }^{18}$

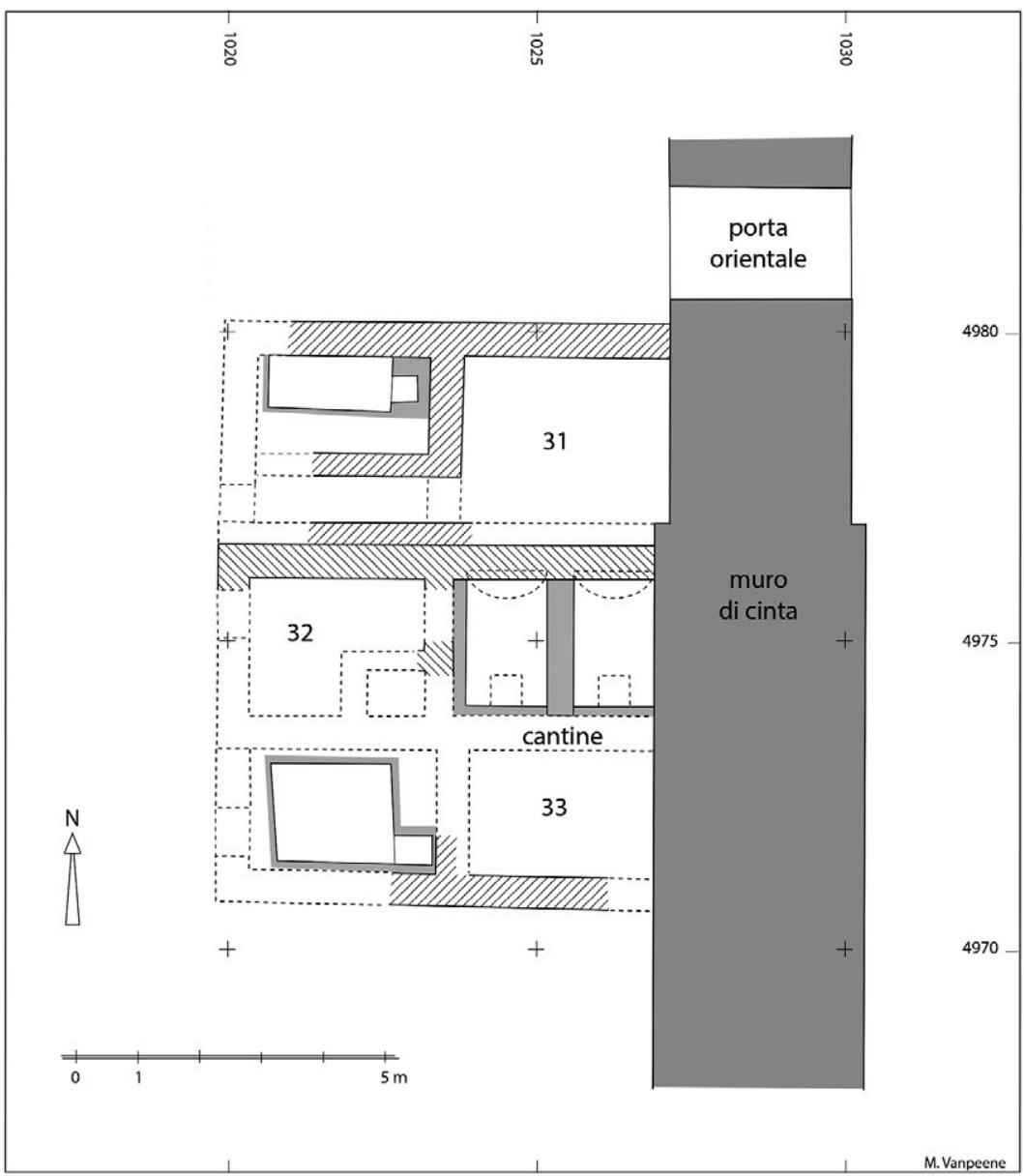

Fig. 3 - Pianta del pastophorion 32 e di quelli adiacenti a nord (31) e a sud (33).

17 Qui e altrove la prima dimensione fornita è quella nord-sud, la seconda quella est-ovest. Per gli edifici si indicano le misure esterne; per i singoli ambienti, che li compongono, si segnalano, invece, quelle interne.

18 Sia la pianta riprodotta a Fig. 3 sia quella stampata a Fig. 7 sono state tracciate dall'architetto Matthieu Vanpeene, da anni membro della Missione. 
Nell'angolo sud-est della camera ad ovest era ubicata una struttura, che oggi è interamente perduta, e che sulla pianta di Franco è rappresentata come un piccolo ambiente di m. 1 x 1,50 con due aperture affrontate, nei lati est ed ovest, ampie all'incirca mezzo metro. L'esiguità delle dimensioni e la larghezza ridotta delle supposte aperture escludono che si trattasse di uno spazio abitativo o di passaggio; parimenti è impossibile che la struttura fosse il supporto di una scala, giacché lo spessore dei muri, così come è riprodotto da Franco, risulta troppo esiguo per sostenere dei gradini. Ė, invece, più probabile che la struttura fosse un silos, di cui le pareti a est e ad ovest erano almeno in parte crollate, tanto che nel disegno di Franco furono rappresentate con aperture. La stanza contenente il silos svolgeva pure la funzione d'ingresso: nella sua parete ovest si apriva una porta, che dava sull'area di passaggio lasciata libera intorno al naos. Essendo il muro per metà perduto e per metà ridotto alle fondamenta, non è rimasta traccia alcuna dell'apertura dell'uscio; però, osservando gli edifici analoghi posti all'interno del peribolo, sembra probabile che la porta d'ingresso fosse posta di fronte a quella interna che immetteva nella seconda camera; quindi doveva trovarsi presso l'estremità settentrionale del muro della stanza. Da questo primo locale si accedeva a quello retrostante ad est, sotto il pavimento del quale erano situate le cantine. Queste sono affiancate l'una all'altra, condividono uno dei lati lunghi e presentano l'asse maggiore orientato in direzione nord-sud, sicché risultano parallele al peribolo limitrofo. Il ripostiglio ad est ha una superficie di m. 2,10 x 1,20 ed un'altezza di m. 1,10; quello ad ovest è ampio m. 2,10 x 1,30 ed alto m. 1,25. Tanto nell'uno quanto nell'altro le volte cominciano $60 \mathrm{~cm}$. al di sopra del pavimento e sono entrambe di tipo nubiano, cioè con archi di mattoni inclinati. Poiché i corsi dei mattoni si appoggiano contro la parete nord, le botole di accesso, necessariamente, dovevano essere collocate dalla parte opposta, vale a dire a sud. Purtroppo di queste non si vedono tracce nei ruderi e non se ne scorgono nemmeno sulla fotografia di Anti, forse perché erano già crollate al momento dello scavo, forse perché furono smantellate per allargarne l'apertura e agevolare il recupero dei papiri ammonticchiati dentro i ripostigli. I resti del pavimento sovrastante le volte appaiono più alti di $70 \mathrm{~cm}$. rispetto alla soglia della porta interna del tempio e al relativo piano di passaggio; sicché si deve ritenere che al momento della costruzione non fu scavato un buco di profondità tale che le due cantine risultassero interamente infossate nel sottosuolo. Come in altri pastophoria del temenos, le cantine in parte scendevano sotterra, in parte sporgevano al di sopra 
del suolo ed erano interrate con materiale di riporto. Essendo state le cantine costruite in questa maniera, si comprende per qual motivo il pavimento posato sopra le volte sia nettamente più alto rispetto al piano di passaggio all'esterno; nello stesso tempo si deve supporre che davanti alla porta d'ingresso del pastophorion fossero installati almeno tre gradini, che permettevano di superare il dislivello e che sono andati completamente perduti con la facciata ovest dell'edificio.

Come si ricava dalla pianta di Franco, su cui le costruzioni del temenos sono rappresentate con colori diversi a seconda della datazione attribuita, sia il pastophorion 32, sia quelli adiacenti a nord e a sud, cioè il 31 e il 33, erano assegnati da Anti al periodo "medio-tolemaico" o al regno di Tolomeo XII, vale a dire agli anni compresi fra l'inizio del II e la metà del I sec. a.C. Essendo tale lasso di tempo piuttosto ampio, è parso opportuno fare il tentativo di precisare le datazioni degli edifici, sfruttando gli indizi ricavabili dal terreno rimasto integro. Con la pulitura di 32, infatti, si era constatato che gli strati sottostanti al piano di passaggio, sia all'interno sia all'esterno della costruzione, non erano troppo alterati, perché gli operai di Anti non li avevano toccati e i saccheggiatori successivi non avevano aperto grossi buchi nel suolo, accanendosi sui muri. Conseguentemente si sono sgombrati anche i resti dei pastophoria 31 e 33 , e si è proceduto allo scavo degli strati conservatisi in posizione intorno e sotto agli edifici. Così si è visto che 32 ed i limitrofi 31 e 33 appoggiavano tutti su uno strato di riporto composto di sabbia, breccia, grumi di argilla, piccoli ciottoli e modiche quantità di alfa e di paglia. Frammisti a questi materiali eterogenei c'erano pure dei cocci e alcune decine di papiri demotici assegnabili al I sec. a.C.; pertanto è apparso evidente che il pastophorion delle cantine ed in due vicini furono eretti negli ultimi decenni del periodo tolemaico, ovvero nella fase iniziale dell'epoca romana.

Successivamente lo scavo è stato spinto al di sotto delle fondamenta degli edifici; e, immediatamente a nord delle cantine, sotto il pastophorion 31, sono affiorati due blocchi di muro accostati, l'uno di seguito all'altro, e paralleli al muro di cinta del tempio, da cui distavano m. 1,90 (Fig. 4). Essi erano fondati nella sabbia vergine e costruiti con grossi mattoni grigi, senza pula nell'impasto, di cm. 38 x 19 x 16; quello a sud era largo m. 3,20 e presentava le assise concave, quello a nord aveva invece un'ampiezza di m. 2,90 e mostrava le assise convesse. Il dispositivo della muratura, l'ampiezza rilevante e l'accostamento dei due blocchi inducevano a pensare che le vestigia apparse fossero un 
tratto di peribolo anteriore a quello scoperto da Grenfell e Hunt, dissepolto in parte da Anti e Bagnani e ben visibile in superficie. ${ }^{19}$

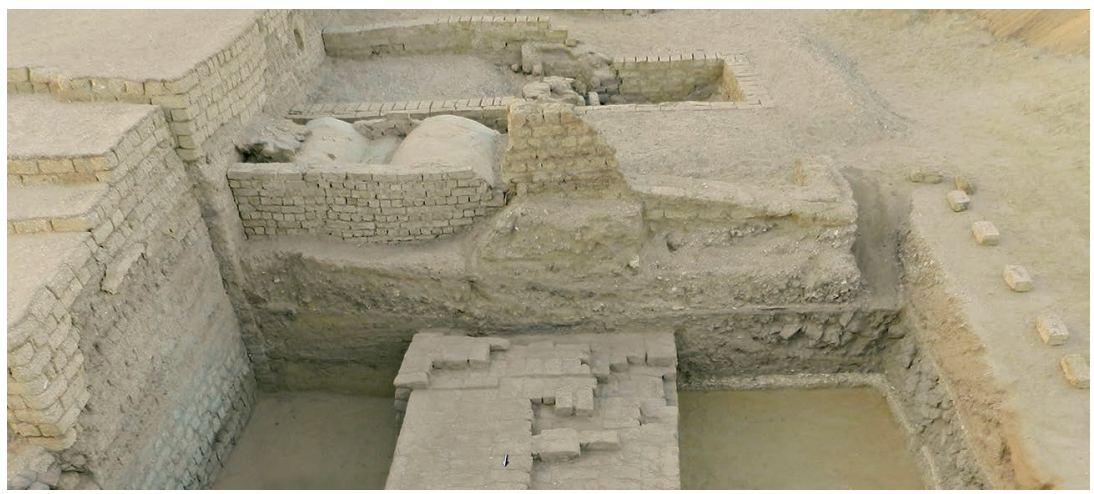

Fig. 4 - Tratto del peribolo più antico apparso a nord delle cantine (in primo piano).

Una conferma di questa impressione, ovviamente, poteva venire solo da una serie di sondaggi eseguiti sull'ipotetico tracciato del muro. Un primo indizio è stato raccolto immediatamente a sud delle due cantine: via via che lo scavo scendeva sotto il pastophorion 33, tornava alla luce il tratto meridionale del blocco con assise concave, che si era incontrato a nord. Altri due segmenti della muraglia sono poi stati localizzati una ventina di metri più a sud, scavando sotto gli edifici contrassegnati con $i$ numeri 37 e 38 sulla pianta di Franco. Nello stesso tempo un quinto pezzo è stato dissepolto presso l'angolo nord-est del peribolo in superficie, sotto l'ambiente indicato come 4D. A quel punto, il tracciato del muraglione poteva essere ricostruito su di un'ottantina di metri; e diventava sempre più attraente l'idea che la struttura scoperta fosse il lato orientale di un peribolo non conosciuto. Sulla base di questa convinzione e con la speranza di individuare il lato sud della costruzione, si sono avviate le indagini nella parte meridionale del temenos, fra i miseri resti dell'ambiente 53 rimesso alla luce nel 1931. Contrariamente alle aspettative, non ci si è imbattuti nei ruderi di una muraglia, ma si è trovata la

19 Sulla struttura delle grandi cinte murarie dei templi si veda J.-C. Golvin - O. Jaubert - El Sayed Hegazy, Essai d'explication des murs «à assises courbes» à propos du grand temple d'Amon-Rề à Karnak, CRAI 134 (1990), 905-946, in partic. 908-939. 
parte inferiore di un forno a calce simile a quelli scoperti negli anni Novanta sulla spianata a est del tempio. ${ }^{20}$ Come palesava il contesto stratigrafico, il forno era stato costruito ed aveva funzionato nel III sec. a.C.; sicché si poteva arguire che, se mai esisteva un peribolo anteriore a quello visibile, il tratto meridionale di esso doveva stare più nord, essendo inverosimile che un forno a calce si trovasse all'interno di un temenos. Le ricerche, quindi, sono state spostate più a nord e, dopo un paio di tentativi rivelatisi infruttuosi, a una quindicina di metri dal lato sud del peribolo scavato da Anti, si è incontrato il tratto est-ovest di quello che si delineava sempre meglio come un muro di cinta più antico rispetto al peribolo conosciuto dal 1899. Un altro paio di sondaggi, effettuati ancora più a nord sotto gli edifici 77 e 16, hanno poi permesso di localizzare due segmenti della muraglia paralleli ai primi individuati ad est (Fig. 5); sicché era ormai sicuro che era stato scoperto un peribolo, di cui si erano messi in luce segmenti di tre lati: a est, a sud e ad ovest.

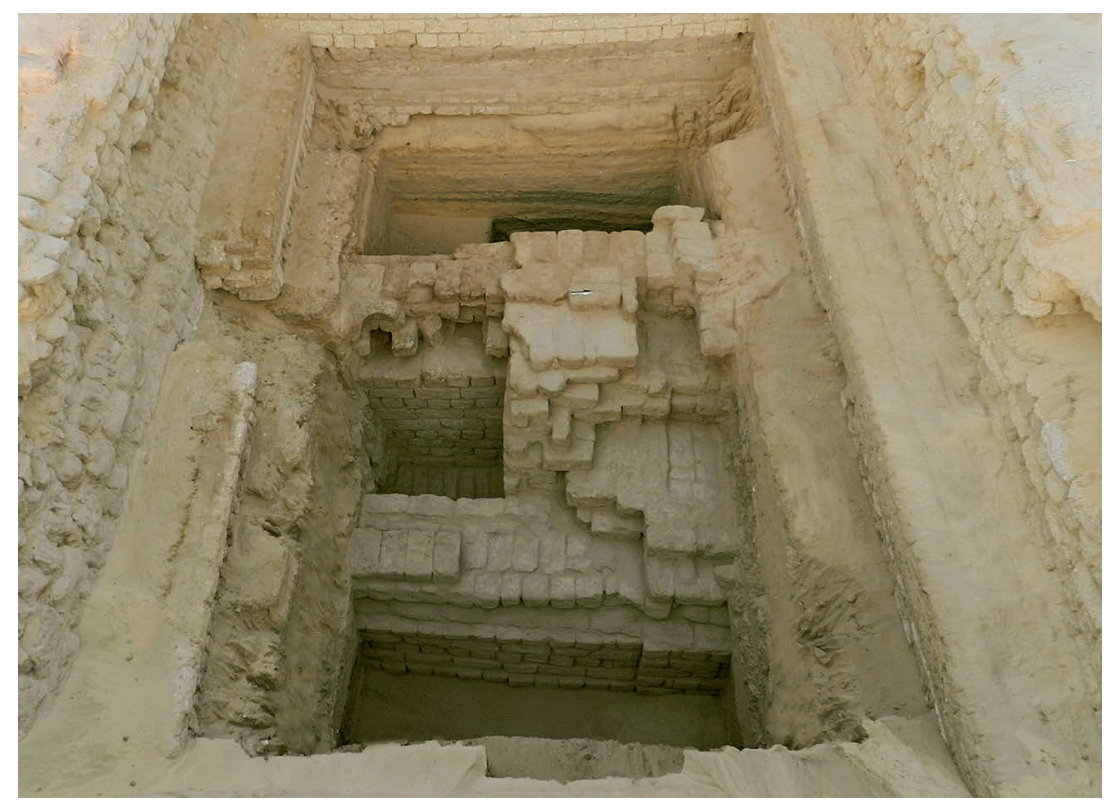

Fig. 5 - Segmento del lato ovest del peribolo più antico sotto l'edificio 16.

20 Cfr. Gallazzi, Lo scavo di una discarica cit. (nt. 3), 192; Id., I lavori a Ummel-Breigât (Tebtynis) degli anni 1997-1999 cit. (nt. 2), 28-29. 
Per trovare il quarto lato, vale a dire quello a nord, non è stato necessario operare altre indagini sul terreno. Nel 2011, infatti, nel corso di lavori di ripulitura a est della porta principale del temenos, era stata trovata la base di un pilone risalente alla fase iniziale del periodo ellenistico; e sul lato opposto dell'entrata, al di sotto delle fondamenta in pietra del portale visibile, era apparsa la parte inferiore di una precedente porta in mattoni coeva del pilone. ${ }^{21}$ Tre anni dopo, nel 2014, effettuando un sondaggio preliminare prima di eseguire dei restauri ad ovest dell'ingresso, si era visto che il blocco del peribolo adiacente alla porta in pietra appoggiava su di un muro più antico fondato nella sabbia vergine (Fig. 6).

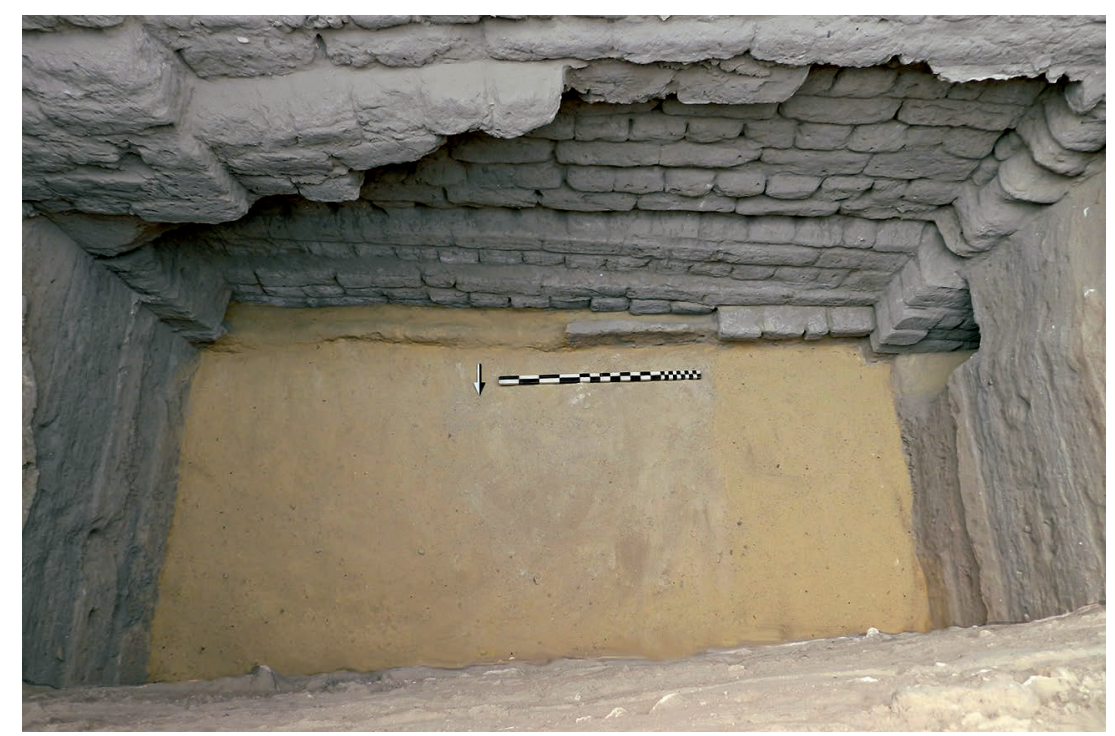

Fig. 6. - Segmento del lato nord del peribolo più antico sottostante al secondo muro di cinta.

Sia nel 2011 sia nel 2014 tutti questi ruderi erano stati interpretati come le vestigia di una prima fase dell'entrata del tempio, che si riteneva modificata più volte fra l'inizio dell'età ellenistica e il principio del-

21 Cfr. Gallazzi, Umm-el-Breigât (Tebtynis). Campagna 2011 cit. (nt. 1), 146148; Gallazzi - Hadji-Minaglou, Umm-el-Breigât (Tebtynis), in Rapport d'activité 20112012 cit. (nt. 8), 73-77. 
l'epoca romana, cioè fra il IV e il I sec. a.C. .22 In verità, il pilone, il portale in mattoni e il blocco di muro sottostante a quello visibile in superficie appartengono anch'essi a quel peribolo di cui si erano scoperti dei tratti a est, a sud e ad ovest: lo prova il fatto che sono fondati al medesimo livello dei pezzi trovati altrove e sono costruiti con mattoni identici. Così, congiungendo i dati raccolti nel 2011 e nel 2014 con quelli forniti dai sondaggi fatti nel corso della campagna, è stato possibile tracciare una pianta del muro di cinta scoperto (Fig. 7). Da questa si vede che la muraglia, rispetto al peribolo scavato da Anti, aveva dimensioni più ridotte, misurando $\mathrm{m} .95 \times 50$ contro $\mathrm{m} .114 \times 60$, e presentava l'asse nord-sud ruotato verso est di poco meno di un grado.

Come è già stato segnalato, in tutti i sondaggi compiuti nel temenos e nelle indagini operate a nord nel 2011 e nel 2014 si è constatato che la muraglia apparsa è fondata nella sabbia vergine e che le trincee delle sue fondamenta non tagliano alcuno strato di occupazione. Da ciò si deduce che la struttura fu eretta all'inizio dell'età tolemaica, perché tutto il settore sud-occidentale dell'insediamento, dove essa si trova, fu urbanizzato a partire dalla fine del IV sec. a.C. ${ }^{23}$ ed il tempio, sicuramente, fu una delle prime costruzioni impiantate. Non è certo un caso, se i livelli di passaggio all'interno del muro di cinta corrispondono a quelli del chiosco scoperto nel 2002 lungo la via processionale, che fu innalzato all'inizio del III sec. a.C. utilizzando mattoni identici a quelli della muraglia. ${ }^{24}$ Pertanto il grande muro altro non è che il peribolo del tempio di Soknebtynis eretto sotto Tolomeo Soter, il nome del quale compare su di un blocco di calcare scolpito, raccolto da Anti nel ' $32 .{ }^{25} \mathrm{~A}$ tale tempio appartengono pure i grossi muri fabbricati con gli stessi mattoni della cinta, che affiorano qua e là nel mezzo del temenos. Anti li aveva dissepolti nel 1932 e li aveva interpretati come avanzi di un tempio faraonico, forse risalente alla XXII dinastia,

22 Cfr. Gallazzi, Umm-el-Breigât (Tebtynis). Campagna 2011 cit. (nt. 1), 146148; Gallazzi - Hadji-Minaglou, Umm-el-Breigât (Tebtynis), in Rapport d'activité 20112012 cit. (nt. 8), 76-77.

23 Cfr. Gallazzi, Umm-el-Breigât (Tebtynis): 2002 cit. (nt. 1), 109; G. HadjiMinaglou, L'apport des Grecs dans l'architecture de la chôra égyptienne: l'exemple de Tebtynis, in P. Ballet (ed.), Grecs et Romains en Égypte, Le Caire 2012, 107-120, in partic. 108.

24 Per il chiosco si rinvia a Gallazzi, Umm-el-Breigât (Tebtynis): 2002 cit. (nt. 1), 108.

25 Cfr. Rondot, Tebtynis, II. Le temple cit. (nt. 6), 86-87. 
perché riteneva che il peribolo da lui portato alla luce risalisse a Tolomeo Soter. ${ }^{26}$ In realtà quelle vestigia non sono ruderi faraonici, perché l'area non fu urbanizzata prima del IV sec. a.C.: sono i resti del naos del santuario eretto sotto Tolomeo Soter congiuntamente alla muraglia, che si è individuata a settembre e ottobre del 2015.

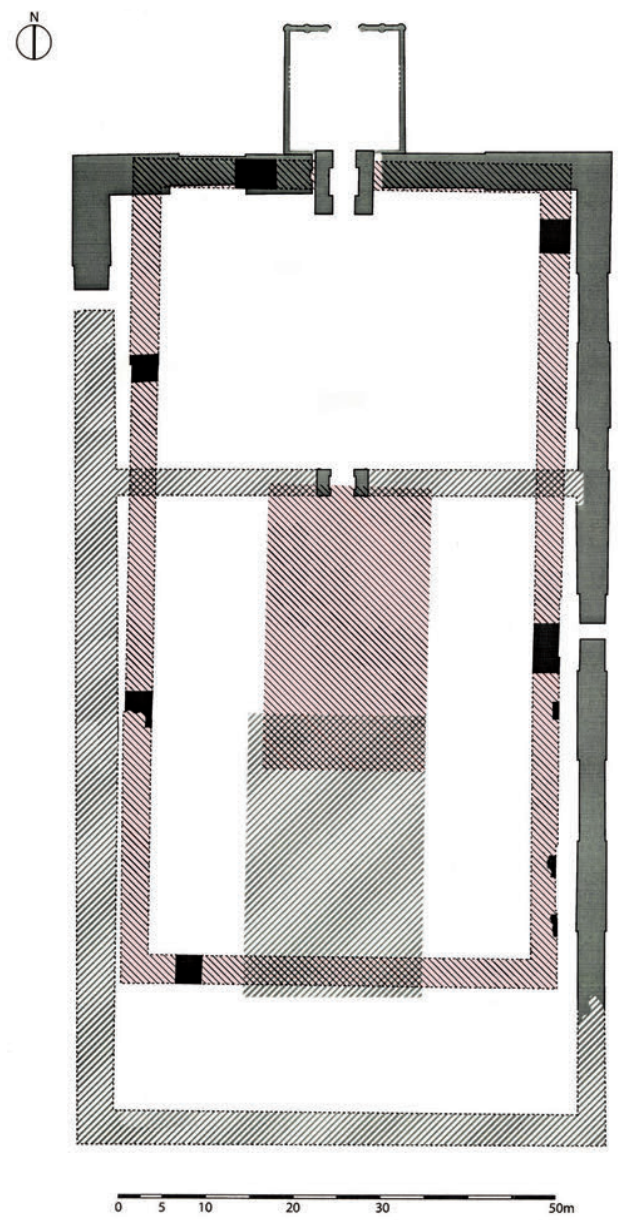

Fig. 7 - Pianta dei due periboli: quello più antico in colore rosso, con $i$ tratti rimessi alla luce evidenziati in colore nero.

26 Cfr. Anti, Scavi a Tebtunis cit. (nt. 6), 474. 
Nei decenni iniziali del I sec. a.C. il peribolo edificato durante il regno di Soter fu sistematicamente demolito e i suoi mattoni furono usati per costruire quello scavato da Anti, che presenta all'interno mattoni recuperati di colore grigio ed all'esterno mattoni nuovi appositamente fabbricati con argilla gialla. Quindi il muro scoperto nel 1899 e dissepolto negli anni Trenta non risale al principio dell'età tolemaica, come si era sempre detto e scritto, ${ }^{27} \mathrm{ma}$ appartiene alla fine di tale periodo, come indicano i cocci ed i papiri del I sec. a.C. rinvenuti nella colmatura delle sue trincee di fondazione e nello strato di riporto steso sui resti della cinta più antica. Contemporaneamente, o pressoché contemporaneamente, al peribolo di Soter, fu raso al suolo anche il naos coevo: i suoi mattoni furono riutilizzati nella nuova muraglia o nei nuovi edifici addossati a questa, mentre gli elementi in pietra in parte, forse, furono recuperati e riadattati, in parte furono distrutti, tanto che Anti ne trovò parecchi pezzi spaccati dentro lo spesso strato di breccia giacente intorno alle fondamenta del nuovo naos. ${ }^{28}$ Purtroppo, i dati al momento disponibili non permettono di stabilire con precisione in che anni siano avvenute la demolizione del primo tempio e la costruzione del secondo. È certo, comunque, che l'erezione del nuovo peribolo fu intrapresa dopo la fine del II sec. a.C. per la ragione indicata or ora, cioè per i materiali del I sec. a.C. presenti nelle colmature. Analogamente è fuor di dubbio che la costruzione della muraglia era compiuta, o quasi compiuta, nel 55 a.C. In quell'anno, infatti, fu innalzata la statua di Tolomeo XII, cui era annessa la dedica datata di SEG XXXIX $1705 .{ }^{29}$ Tale statua, come Anti precisa nel suo taccuino, fu trovata rotta in quattro pezzi,

27 Cfr. Anti, Gli scavi della Missione cit. (nt. 6), 390; G. Bagnani, Gli scavi di Tebtunis, Bollettino d'Arte 27. III (1933), 119-134, in partic. 119; É. Bernand, Recueil d'inscriptions grecques du Fayoum, III. La «méris» de Polémôn, Le Caire 1981, 12; P. Davoli, L'archeologia urbana nel Fayyum di età ellenistica e romana, Napoli 1998, 198; Gallazzi, Umm-el-Breigât (Tebtynis). Campagna 2011 cit. (nt. 1), 147-148; Gallazzi - HadjiMinaglou, Umm-el-Breigât (Tebtynis), in Rapport d'activité 2011-2012 cit. (nt. 8), 76.

28 Del recupero dei pezzi del primo naos Anti parla più volte nelle pagine del suo taccuino, dove li considera avanzi del tempio faraonico, che egli pensava esistesse nell'area.

29 Per la statua e per la dedica si vedano G. Bastianini - C. Gallazzi, Un'iscrizione inedita di Tebtynis per una statua controversa di Tolomeo XII, NAC 18 (1989), 201-209; Gallazzi, Umm-el-Breigât (Tebtynis). Campagna 2011 cit. (nt. 1), 146; Gallazzi - Hadji-Minaglou, Umm-el-Breigât (Tebtynis), in Rapport d'activité 2011-2012 cit. (nt. 8), 73. 
due dei quali erano deposti presso la base situata davanti all'ingresso del vestibolo del tempio, sul lato orientale. Il blocco iscritto con la dedica stava, invece, fra le pietre accatastate sul lato opposto dell'entrata, vale a dire ad ovest. Come si legge nel quaderno di Anti, il plinto della statua si adatta perfettamente alla base rimasta in situ; e con le dimensioni di quest'ultima si accordano pure le misure del blocco della dedica. Si deve, quindi, concludere che nel 55 a.C., come dice l'iscrizione, la statua di Tolomeo XII si ergeva davanti al vestibolo del tempio. Siccome il vestibolo, sul lato sud, è appoggiato al peribolo più recente, è manifesto che la costruzione di quest'ultimo deve essere anteriore al 55 a.C. Pertanto il nuovo muro di cinta, se fu eretto dopo il II sec. e prima del 55 a.C., potrebbe essere stato costruito sotto Tolomeo X, che rimase sul trono sino all' 88 a.C., ovvero sotto Tolomeo IX, che riprese il potere nello stesso anno, o sotto Tolomeo XII, che ascese al trono nell'81 a.C., rimanendovi per trent'anni. La prima delle tre possibilità ammissibili è, però, poco probabile, infatti i materiali del I sec. a.C. presenti nelle colmature risultano troppo abbondanti, perché si possa ritenere che i riempimenti siano stati fatti proprio nel decennio iniziale del secolo. Sembra, invece, più verosimile che la nuova muraglia sia stata eretta sotto Tolomeo IX, oppure sotto Tolomeo XII, ovvero in parte sotto l'uno e in parte sotto l'altro re, tanto più che si conoscono molti lavori di costruzione e di restauro di templi effettuati in varie aree dell'Egitto sotto i due sovrani. ${ }^{30}$

Per il momento non si può dire di più sull'erezione del secondo santuario dopo la demolizione di quello innalzato sotto Tolomeo Soter; ma non è escluso che la cronologia dei lavori possa essere precisata via via che le indagini saranno estese all'interno del temenos. In tale area, infatti, gli strati anteriori all'età romana si presentano ancora in buona parte intatti e potrebbero contenere parecchio materiale utile per conoscere meglio l'evoluzione del complesso. Lo si è constatato durante i lavori realizzati nel 2015, allorché si è riportata alla luce una discreta quantità di ceramica, in prevalenza frammentaria, ma

30 Poiché sarebbe troppo lungo enumerare in una nota tutti gli interventi effettuati sotto Tolomeo IX e sotto Tolomeo XII, si rinvia chi desideri avere informazioni dettagliate a W. Huss, Der makedonische König und die ägyptischen Priester, Stuttgart 1994, 35-37; Id., Ägypten in hellenistischen Zeit. 332-30 v. Chr., München 2001, 701-702. 
sempre utile per datare gli strati, e si sono pure ritrovati degli oggetti integri e talvolta fuori dell'ordinario. Basti ricordare che lo strato di riporto steso sui ruderi del primo peribolo ha restituito legni intarsiati e incrostati di smalti, simili a quelli rinvenuti nella discarica a est del santuario negli anni 1996, 1998 e $2000 ;{ }^{31}$ ha dato un frustino intatto, fabbricato con canne di palude avvolte in fibre di papiro, ${ }^{32}$ e ha fornito una statuetta di coccodrillo in steatite, che costituiva il deposito di fondazione del pastophorion con le cantine (Fig. 8). ${ }^{33}$

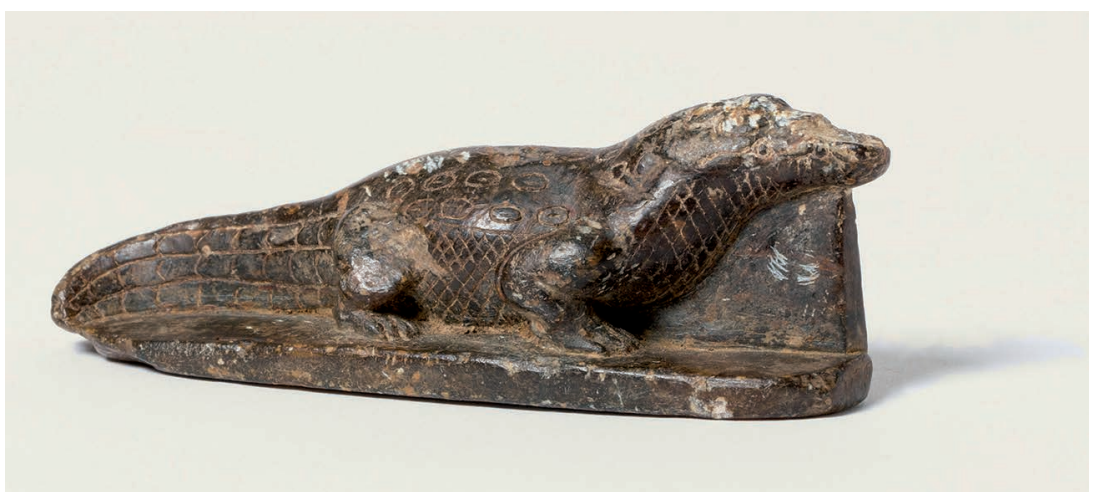

Fig. 8 - Statuetta di coccodrillo in steatite.

31 Cf. Rondot, Tebtynis, II. Le temple cit. (nt. 6), 260, fig. 68 ; Gallazzi, I lavori cit. (nt. 2), 29; Mathieu, Travaux de l'Institut français d'archéologie orientale en 20002001 cit. (nt. 3), 552.

32 Il pezzo è identico a un esemplare raccolto nella vicina discarica durante la campagna del 2002, per il quale cfr. Gallazzi, Umm-el-Breigât (Tebtynis): 2002 cit. (nt. 1), 114.

33 Non si conoscono statuette in steatite, che raffigurino un coccodrillo adagiato su di una piccola base, con la testa un poco sollevata e la coda arcuata verso destra, al pari di quella rinvenuta. Tuttavia, lo stesso tipo di rappresentazione si trova, ex. gr. in un esemplare in basalto nero conservato al Museo Egizio di Torino (con una mortasa nella testa per l'installazione di un disco solare o di un ureo; Nuovo regno?; cfr. C. Dolzani, Il dio Sobk, in Atti della Accademia nazionale dei Lincei. Memorie. Classe di Scienze morali, Serie VIII, vol. X. 4, Roma 1961, 163-269, in partic. 205, Tav. IX, Fig. 1), in un bronzo del Louvre (con ureo e disco solare; Bassa epoca; cfr. Coptos. L'Égypte antique aux portes du désert. Lyon, musée des Beaux-Arts, 3 février - 7 mai 2000, Lyon - Paris 2000, 110, nr. 79) ed in un pezzo in granito grigio del Musée du Petit Palais (58 a.C.; cfr. La gloire d'Alexandrie. Paris, 7 mai - 26 juillet 1998, Paris 1998, 197, nr. 142). 
Inoltre, in due dei sondaggi sono stati rinvenuti nidi di topi fabbricati con fuscelli, steli di paglia e pezzi di papiro (Fig. 9). Questi ulti$\mathrm{mi}$, naturalmente, erano in genere assai ridotti, essendo stati triturati dalle bestie; ma, insieme a minuscoli coriandoli di pochi millimetri quadrati, c'erano pure pezzi più ampi, con testi in ieratico, demotico e greco, sufficientemente interessanti da richiedere una pubblicazione. ${ }^{34}$ Ai papiri estratti dai nidi dei topi se ne sono aggiunti alcuni altri con documenti in greco ed in demotico, che sono affiorati in vari punti del temenos. E la messe dei testi è stata completata da 70 domande oracolari demotiche del I sec. a.C., che giacevano sotto il pastophorion delle cantine e sotto quello limitrofo a nord, sovente ancora arrotolate e munite di sigillo (Fig. 10). ${ }^{35}$

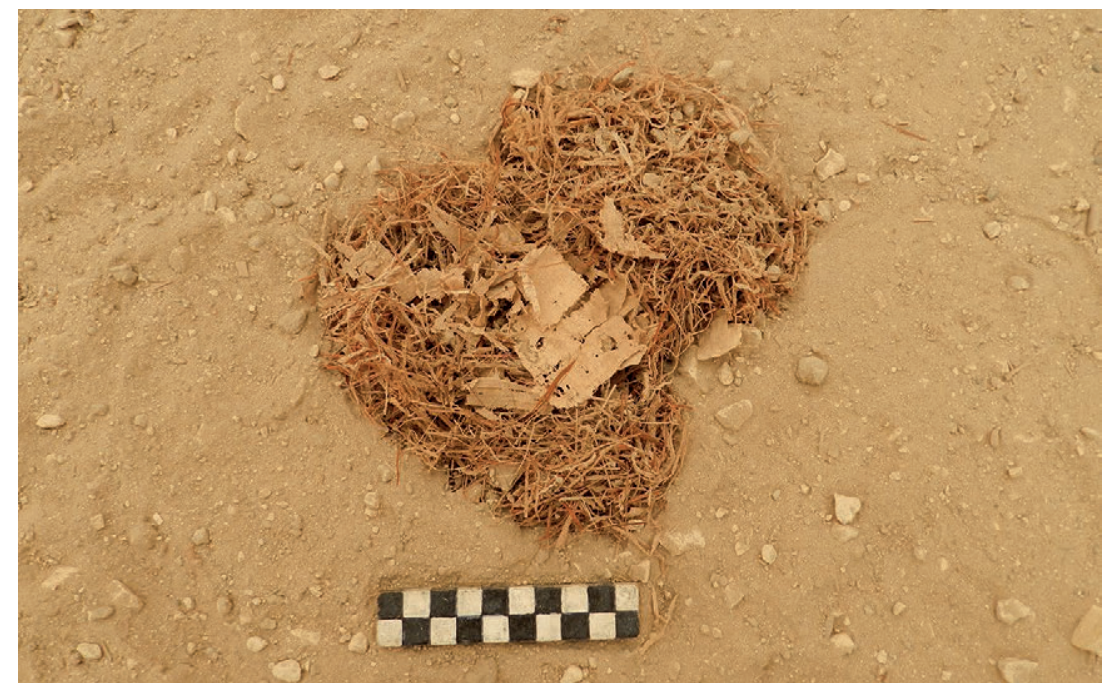

Fig. 9 - Nido di topi contenente papiri.

34 Una trattazione dettagliata del ritrovamento dei papiri nelle tane dei topi è reperibile in C. Gallazzi, Una maniera inconsueta di recuperare i papiri, APF 62 (2016), 151-161.

35 Circa 300 biglietti analoghi, ma risalenti al III sec. a.C. ed in parte scritti in greco, sono stati raccolti a pochi metri di distanza, al di là del muro di cinta del temenos, nel 1997 e nel 2007, come si dice in C. Gallazzi, Le 300 nuove domande oracolari di Tebtynis, in A. Gasse - F. Servajean - Ch. Thiers (éd.), Et in Aegypto et ad Aegyptum. Recueil d'études dédiées à Jean-Claude Grenier, II, Montpellier 2012, 331-344. 


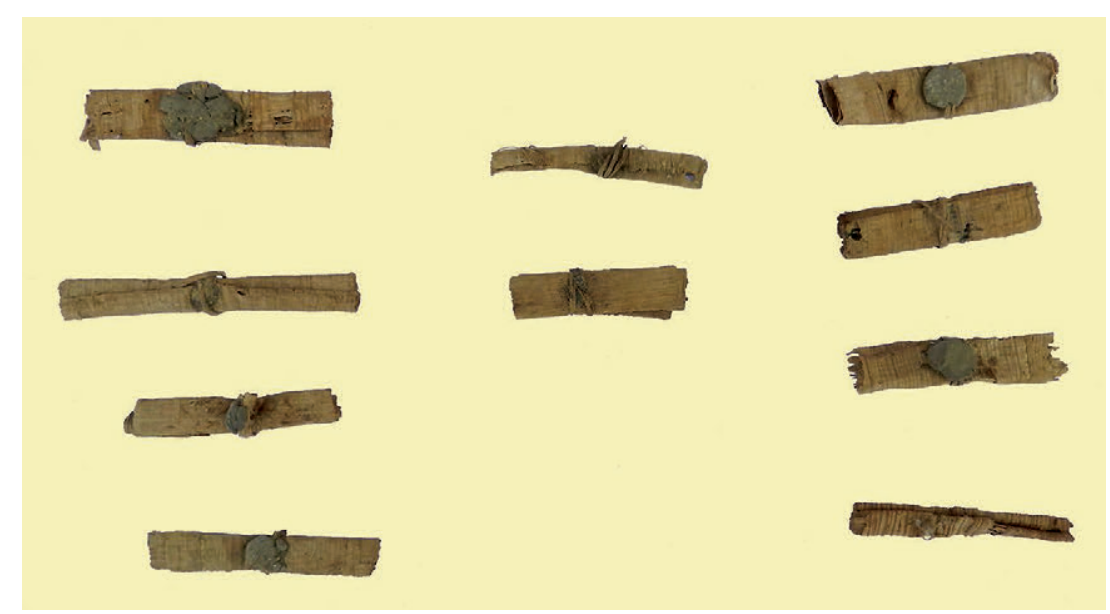

Fig. 10 - Domande oracolari arrotolate e sigillate.

Visti i risultati raccolti, dopo che si era cominciato a lavorare nel tempio di Soknebtynis con il solo intento di ripulire e di fotografare due cantine già scavate, non è arrischiato ritenere che il vasto complesso, scoperto più di un secolo addietro, possa racchiudere ancora qualche sorpresa, forse pure eclatante. 
